\title{
Seizure and neuropsychological outcomes in a large series of selective amygdalohippocampectomies with a minimally invasive subtemporal approach
}

\author{
*Alexander C. Whiting, MD, Tsinsue Chen, MD, Kyle I. Swanson, MD, Corey T. Walker, MD, \\ Jakub Godzik, MD, Joshua S. Catapano, MD, and Kris A. Smith, MD \\ Department of Neurosurgery, Barrow Neurological Institute, St. Joseph's Hospital and Medical Center, Phoenix, Arizona
}

OBJECTIVE Debate continues over proper surgical treatment for mesial temporal lobe epilepsy (MTLE). Few large comprehensive studies exist that have examined outcomes for the subtemporal selective amygdalohippocampectomy (sSAH) approach. This study describes a minimally invasive technique for sSAH and examines seizure and neuropsychological outcomes in a large series of patients who underwent SSAH for MTLE.

METHODS Data for 152 patients (94 women, 61.8\%; 58 men, 38.2\%) who underwent sSAH performed by a single surgeon were retrospectively reviewed. The SSAH technique involves a small, minimally invasive opening and preserves the anterolateral temporal lobe and the temporal stem.

RESULTS All patients in the study had at least 1 year of follow-up (mean [SD] 4.52 [2.57] years), of whom $57.9 \%$ (88/152) had Engel class I seizure outcomes. Of the patients with at least 2 years of follow-up (mean [SD] 5.2 [2.36] years), 56.5\% (70/124) had Engel class I seizure outcomes. Preoperative and postoperative neuropsychological test results indicated no significant change in intelligence, verbal comprehension, perceptual reasoning, attention and processing, cognitive flexibility, visuospatial memory, or mood. There was a significant change in word retrieval regardless of the side of surgery and a significant change in verbal memory in patients who underwent dominant-side resection $(p<$ 0.05). Complication rates were low, with a $1.3 \%(2 / 152)$ permanent morbidity rate and $0.0 \%$ mortality rate.

CONCLUSIONS This study reports a large series of patients who have undergone SSAH, with a comprehensive presentation of a minimally invasive technique. The sSAH approach described in this study appears to be a safe, effective, minimally invasive technique for the treatment of MTLE.

https://thejns.org/doi/abs/10.3171/2020.3.JNS192589

KEYWORDS epileptology; functional neurosurgery; mesial temporal lobe epilepsy; selective amygdalohippocampectomy; subtemporal

$\mathrm{N}$ EUROSURGICAL intervention for mesial temporal lobe epilepsy (MTLE) in rigorously screened patients is a proven, efficacious treatment. ${ }^{1}$ Surgical removal of the mesial temporal structures in these patients is associated with long-term seizure control in $60 \%-70 \%$ of patients., ${ }^{1,2}$ Despite the significant preoperative evaluation needed to select the appropriate patients for this procedure, the benefits from high rates of seizure freedom have proven to be overtly cost-effective in the long term..$^{3-5}$ Even with a consensus on the efficacy of surgical interven- tion, significant disagreement remains regarding the type of surgical approach that should be used.

A complete anterior temporal lobectomy (ATL) was the traditional standard of care. Recently, however, many "cortical-sparing" techniques, including selective amygdalohippocampectomy and laser interstitial thermal therapy, have been evaluated. ${ }^{2,6,7}$ Open surgical approaches to perform a selective amygdalohippocampectomy include transsylvian, inferior temporal gyrus, and subtemporal approaches to perform a similar deep resection of epilep-

ABBREVIATIONS ATL = anterior temporal lobectomy; BDI-II = Beck Depression Inventory-II; BNT = Boston Naming Test; BVMT-R = Brief Visuospatial Memory TestRevised; MTLE = mesial temporal lobe epilepsy; MTS = mesial temporal sclerosis; RAVLT = Rey Auditory Verbal Learning Test; SLAH = stereotactic laser amygdalohippocampectomy; $\mathrm{SSAH}$ = subtemporal selective amygdalohippocampectomy; TMT = Trail Making Test.

SUBMITTED September 19, 2019. ACCEPTED March 30, 2020.

INCLUDE WHEN CITING Published online June 12, 2020; DOI: 10.3171/2020.3.JNS192589.

${ }^{*}$ A.C.W. and T.C. contributed equally to this work and share first authorship. 
togenic tissue ${ }^{8,9}$ Despite the variety of approaches used by different surgeons, literature that specifically evaluates outcomes in patients who have undergone subtemporal selective amygdalohippocampectomy (sSAH) has lagged behind literature describing other approaches. At our institution, $\mathrm{sSAH}$ is preferred because of its minimally invasive nature and ability to preserve the anterolateral temporal lobe and temporal stem. The goal of this study was to investigate the epileptological and neuropsychological outcomes of a large cohort of patients who underwent sSAH for refractory MTLE at a high-volume surgical center.

\section{Methods}

\section{Patient Population and Data Collection}

This study was conducted with the approval of the St. Joseph's Hospital and Medical Center Institutional Review Board for Human Research in Phoenix, Arizona. The medical records of all patients who underwent an sSAH by the senior author (K.A.S.) between April 2004 and April 2017 were retrospectively reviewed. The records of patients with medically refractory epilepsy who had undergone a significant epilepsy neurology evaluation and were recommended for resection of the amygdala and hippocampus were included in the study. Patients were required to have had at least 1 year of clinical follow-up to be evaluated. Exclusion criteria included patients who had additional resective or disconnective surgery performed at the time of the sSAH, patients who had undergone prior resective epilepsy surgery, and patients with a neoplastic diagnosis.

Data for included patients were retrospectively reviewed for seizure outcomes, complications, preoperative characteristics, and patient demographic characteristics. A total of 152 patients met inclusion criteria and were reviewed regarding sex, side of surgery, age, MRI findings, pathology findings, and preoperative workup. Preoperative MRI was evaluated for evidence of mesial temporal sclerosis (MTS) based on review by a neuroradiologist. Patients were classified as "skip" candidates or "nonskip" candidates. Skip candidates had a noninvasive preoperative workup that was deemed convincing enough to warrant proceeding with the sSAH without invasive monitoring by the hospital's multidisciplinary epilepsy team. Frequently, skip candidates demonstrated concordance of noninvasive data, including a mesial temporal semiology, evidence of MTS on MRI, and convincing evidence for lateralization. Nonskip candidates were deemed by the hospital's multidisciplinary epilepsy team to be lacking enough concordant data to proceed with surgery directly and required invasive intracranial monitoring to confirm the correct procedure before proceeding with the surgery. Pathology specimens were reviewed by a neuropathologist and classified as either demonstrating hippocampal sclerosis or not.

\section{Seizure and Neuropsychological Outcomes}

Patients were evaluated at regular intervals according to the Engel classification regarding seizure outcome after surgery. ${ }^{10}$ Postoperatively, patients were considered Engel class I if they were free of disabling seizures, class II if they had only rare disabling seizures, class III if they demonstrated worthwhile improvement in seizure frequency, and class IV if they demonstrated no worthwhile improvement or were worse. Patients were also evaluated on the basis of freedom from antiepileptic drugs after surgery. For this study, the seizure outcome evaluations at 1 year and any evaluation annually thereafter were retrospectively reviewed. All patients underwent a standardized battery of neuropsychological and neurocognitive tests administered by a trained neuropsychologist before surgery. Patients were evaluated on the basis of intelligence (fullscale IQ), attention and processing speed (Trail Making Test [TMT] part A), comprehension and reasoning (Verbal Comprehension Index and Perceptual Reasoning Index of the Wechsler Adult Intelligence Scale, 4th Edition), cognitive flexibility (TMT part B), visuospatial memory (Brief Visuospatial Memory Test-Revised [BVMT-R]), verbal memory (Rey Auditory Verbal Learning Test [RAVLT]), word retrieval (Boston Naming Test [BNT]), and mood (Beck Depression Inventory-II [BDI-II]). All patients who were willing and able to perform 6-month neuropsychological and neurocognitive follow-up testing were evaluated with the same tests before and after surgery, and the data were retrospectively reviewed.

\section{Complications}

All patients who underwent sSAH and met the inclusion criteria, regardless of time or consistency of followup, were analyzed for postoperative morbidity and mortality. All patients underwent a neurological examination before surgery and were evaluated immediately after the operation and at all subsequent follow-up appointments, including bedside visual field confrontation testing. In addition, all patients were analyzed for postoperative complications.

\section{Statistical Analysis}

Differences between variables within the subgroup of patients who demonstrated Engel class I seizure outcomes, including invasive monitoring, sex, side of surgery, presence of MTS on MRI, and pathologic findings, were analyzed using odds ratios and 95\% confidence intervals. Mean group changes in neuropsychological test results and outcomes were statistically analyzed using two-tailed paired Student t-tests. For all analyses, $\mathrm{p}<0.05$ was considered to be statistically significant. ${ }^{11,12}$

\section{Surgical Procedure}

All included patients underwent sSAH performed by the senior author (K.A.S.). The operative technique was originally detailed by Little et al., ${ }^{13}$ but it has since been modified. Patients are positioned supine, with a large bump under the ipsilateral shoulder and the head turned contralaterally $90^{\circ}$. The vertex is tilted down slightly, so the zygoma is the highest point on the field. The table can be turned, and a large shoulder bump should be used to minimize jugular venous pressure in the neck during positioning. A small 3- to 4-cm curvilinear incision is made from $1 \mathrm{~cm}$ anterior to the tragus with the tail curving pos- 

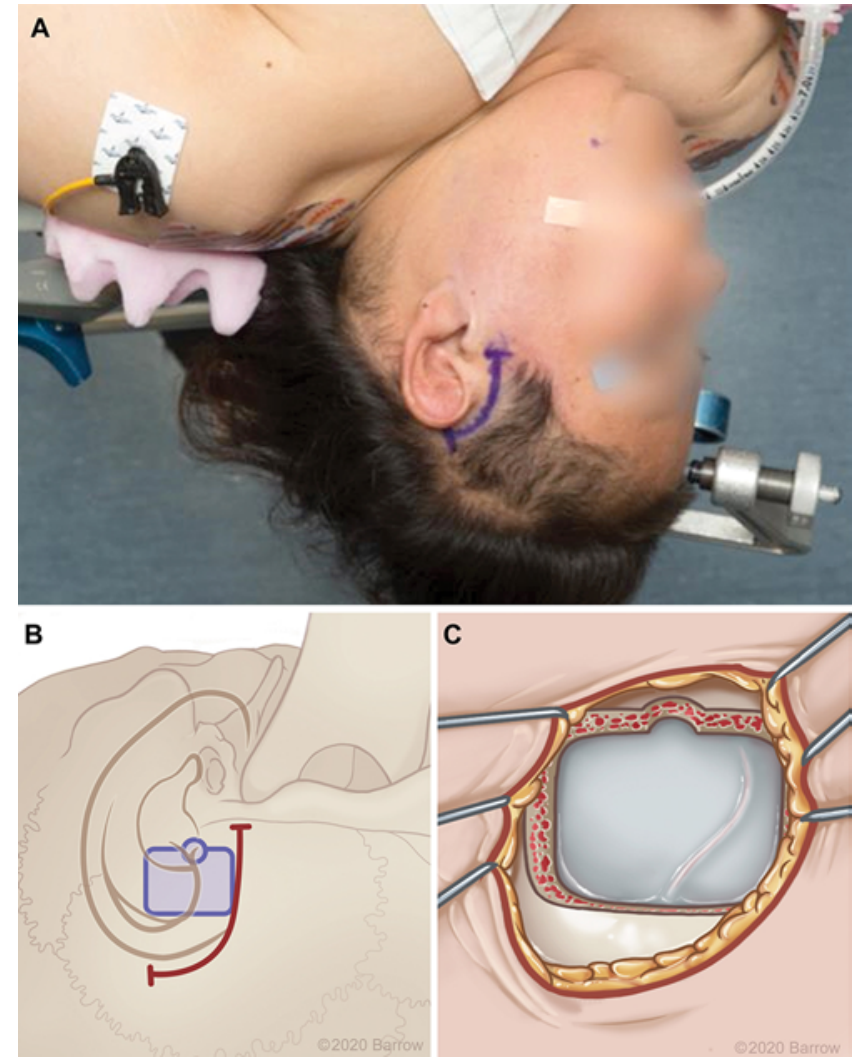

FIG. 1. A: Photograph illustrative of patient head position with the incision marked (purple) for left-sided surgery. B: Illustration of craniotomy location (blue area) in relation to skin incision (red line). C: Illustration of craniotomy appearance, with the bone flap removed and fishhooks in place. Used with permission from Barrow Neurological Institute, Phoenix, Arizona. Figure is available in color online only.

teriorly over the ear (Fig. 1A and B). It is necessary to shave only a small portion of the patient's hair, if any at all. A 2-cm temporal craniotomy is created at the floor of the middle fossa with the use of fishhooks for retraction (Fig. 1C). The dura is carefully opened in a C-shaped fashion and retracted inferiorly. Subtemporal cerebrospinal fluid egress and mannitol administration are used to facilitate brain relaxation such that a fixed retractor is not needed to allow access to the subtemporal region. Care must be taken to avoid excessive temporal lobe retraction, which can cause cortical injury or venous avulsion.

Once adequate subtemporal exposure is achieved, a stepwise "keyhole" technique is used to remove the presumed epileptogenic structures through the small craniotomy (Fig. 2). First, the collateral sulcus and fusiform gyrus are identified. With image guidance, the surgeon enters the collateral sulcus on a trajectory toward the anterior tip of the temporal lateral ventricle horn. Once the temporal tip is entered, cerebrospinal fluid egress facilitates additional working room and allows identification of crucial landmarks. The choroid plexus is used to identify the choroidal fissure, with identification of the amygdala anterosuperior to this landmark. Image guidance is used to verify the location, and the amygdala is then debulked via ultrasonic aspiration and suction. Once the pia is iden- tified, careful subpial dissection is used to remove the remaining rim of the amygdala and uncus. The internal carotid artery, posterior communicating artery, oculomotor nerve, and tentorial edge are visible through the undisrupted pia-arachnoid layer.

The parahippocampal gyrus is then debulked to create room to roll the hippocampal structures inferiorly. The hippocampus is then carefully separated from the choroidal fissure using dissection in the inferior direction. A posterior coronal cut is then made in the hippocampus using the ultrasonic aspirator and subpial dissection. The hippocampus is then disconnected systematically from its medial arterial feeders in the hippocampal fissure, with the vessels cauterized and divided as distally as possible to avoid damaging cisternal vessels feeding other structures. The disconnected hippocampus is then removed en bloc. Finally, with the use of image guidance, the hippocampal tail is resected posteriorly to the level of the collicular plate. The complete resection can be performed through a keyhole craniotomy with minimal disruption of the anterolateral temporal lobe and the temporal stem. Video 1 comprehensively demonstrates this minimally invasive technique.

VIDEO 1. Operative video demonstrating the sSAH technique. Used with permission from Barrow Neurological Institute, Phoenix, Arizona. Click here to view.

\section{Results}

\section{Demographics and Seizure Outcomes}

Demographic characteristics of the 152 patients who underwent sSAH with at least 1 year of follow-up were included. The mean (SD) clinical follow-up was 4.52 (2.57) years (range 1.04-12.37 years). Of the 152 patients, $90(59.2 \%)$ patients had findings of MTS on MRI, $141(92.8 \%)$ patients had gross pathology specimens with findings consistent with hippocampal sclerosis, and 89 $(58.6 \%)$ patients were skip candidates and proceeded to surgery, without invasive intracranial monitoring (Table 1). Engel classification seizure outcomes in patients with at least 1 year of follow-up are given in Table 2:10 88 (57.9\%) patients, $39(25.7 \%)$ patients, $15(9.9 \%)$ patients, and 10 (6.6\%) patients demonstrated Engel class I, II, III, and IV seizure outcomes, respectively. Twenty-three (15.1\%) patients with at least 1 year of follow-up did not receive any antiepileptic drugs; all of these patients were classified as Engel class I. Of all patients, those in Engel classes I and II, $127(83.6 \%)$, accounted for the vast majority of patients.

A total of 124 patients had at least 2 years of clinical follow-up. These patients had a mean (SD) clinical followup of 5.20 (2.36) years (range 2.00-12.37 years). Of these patients, $70(56.5 \%)$ had Engel class I seizure outcome, 33 (26.6\%) had Engel class II seizure outcome, 13 (10.5\%) had Engel class III seizure outcome, and 8 (6.5\%) had Engel class IV seizure outcome (Table 3). Additionally, 22 $(17.7 \%)$ patients with at least 2 years of follow-up did not receive any antiepileptic drugs; all of these patients were classified as Engel class I (Table 3).

A statistical analysis of patient data was performed comparing preoperative variables within the subgroup of patients with at least 1 year of follow-up who had Engel 
A

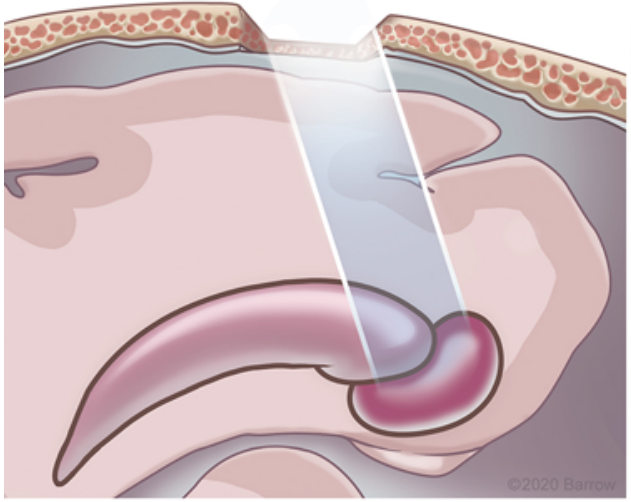

C

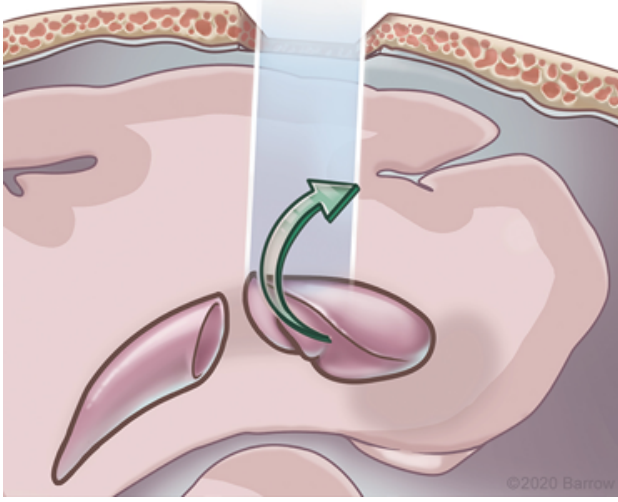

B

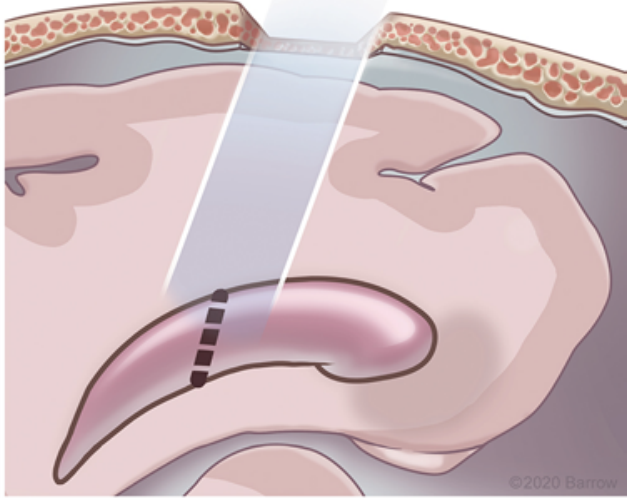

D

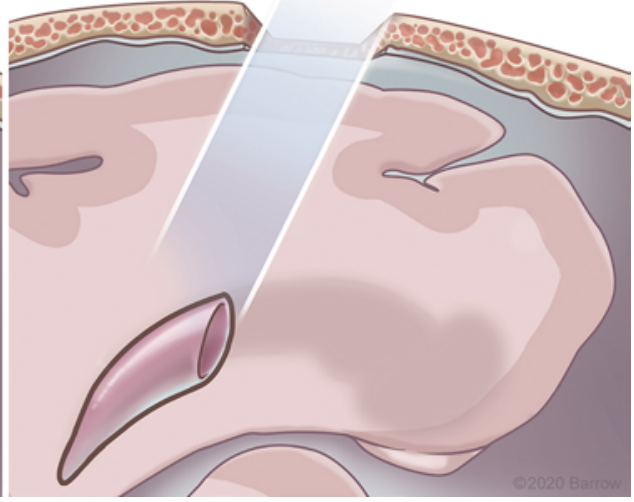

FIG. 2. Illustration of steps for the minimally invasive "keyhole" approach to an SSAH. A: With the use of the anterior temporal tip as a landmark, the amygdala is resected completely. $\mathrm{B}$ : A posterior coronal cut (dashed line) is made down to the pia through the hippocampus. C: The hippocampus is separated from the choroidal fissure and then rolled forward and inferiorly, with coagulation and dissection of the arterial feeders arising from the hippocampal fissure. The hippocampus is then removed en bloc. D: The hippocampal tail is chased posteriorly to the level of the collicular plate and then resected. Used with permission from Barrow Neurological Institute, Phoenix, Arizona. Figure is available in color online only.

class I seizure outcomes (Table 4). Sex, side of surgery, and final pathology findings of hippocampal sclerosis were not significantly associated with Engel class I seizure outcomes. Patients who were deemed skip candidates and did not require invasive intracranial monitoring before surgery had a significant difference in Engel class I seizure outcomes compared with nonskip patients $(\mathrm{p}=0.005)$. Of the 89 skip patients with at least 1 year of follow-up, 60 (67.4\%) had Engel class I outcomes, whereas, of the 63 nonskip patients, 28 (44\%) had Engel class I outcomes. The finding of MTS on preoperative MRI was also significantly associated with Engel class I outcomes $(\mathrm{p}=0.017)$. Of the 90 patients with MTS who had at least 1 year of follow-up, 59 (66\%) had Engel class I outcomes, whereas of the 61 patients without preoperative findings of MTS on MRI, only 28 (46\%) had Engel class I outcomes.

\section{Neuropsychological Outcome}

Of the 152 patients with at least 1 year of clinical follow-up, 38 (25\%) completed both preoperative and postoperative neuropsychological testing by a neuropsychologist. Patients underwent tests that included full-scale IQ, TMT part A, TMT part B, Verbal Comprehension Index, Per- ceptual Reasoning Index, BVMT-R, RAVLT-short delay, BNT, and BDI-II. Of the 38 patients, 19 (50\%) underwent surgery on the dominant hemisphere, and 19 (50\%) underwent surgery on the nondominant hemisphere. The mean differences in preoperative and postoperative scores were statistically compared using two-tailed paired Student ttests (Table 5). Of note, the mean difference in scores was statistically significant in the dominant-hemisphere cohort for the RAVLT-short delay test $(\mathrm{p}=0.02)$ and the BNT ( $\mathrm{p}$ $=0.04)$. The mean difference in scores was statistically significant in the nondominant cohort only with the BNT $(\mathrm{p}=0.04)$. Many patients were unable to complete both preoperative and postoperative neuropsychological testing for a variety of reasons, including challenges with insurance funding of postoperative neuropsychological testing, patient time constraints, travel for out-of-town patients, and lack of social support for others to make multiple medical follow-up visits.

\section{Complications}

All patients underwent a neurological examination before surgery and were evaluated immediately after the operation and at all subsequent follow-up appointments. Of 
TABLE 1. Demographic and clinical characteristics of patients who underwent SSAH with at least 1 year of follow-up

\begin{tabular}{lc}
\hline \multicolumn{1}{c}{ Variable } & Value \\
\hline No. of patients & 152 \\
\hline Mean duration of follow-up (range) & $1649(379-4519)$ \\
\hline Days & $4.52(1.04-12.38)$ \\
\hline Yrs & $58(38.2)$ \\
\hline Sex & $94(61.8)$ \\
\hline Male & $73(48.0)$ \\
\hline Female & $79(52.0)$ \\
\hline Side of op & $40.6(11-73)$ \\
\hline Rt & \\
\hline Lt & $90(59.2)$ \\
\hline Mean age (range), yrs & $61(40.1)$ \\
\hline MTS findings on MRI & $1(0.7)$ \\
\hline Positive & $89(58.6)$ \\
\hline Negative & $63(41.4)$ \\
\hline Unknown & \\
\hline Invasive intracranial monitoring* & $141(92.8)$ \\
\hline No (skip group) & $3(2.0)$ \\
\hline Yes (nonskip group) & \\
\hline Hippocampal sclerosis pathology findings & \\
\hline Positive & \\
\hline Negative & \\
\hline Unknown (pathology findings not available) & \\
\hline
\end{tabular}

Values are presented as the number of patients (\%) unless stated otherwise.

* Patients in the skip group did not undergo invasive intracranial monitoring

before surgery, whereas those in the nonskip group did.

the 152 patients, only $8(5.3 \%)$ experienced postoperative complications; 6 (3.9\%) patients had complications classified as asymptomatic or a transient neurological deficit that had resolved by the last follow-up. These complications included 1) a nonoperative, asymptomatic subdural hematoma; 2) a nonoperative, asymptomatic epidural hematoma; 3) blood in the resection cavity with seizures on postoperative day 1 ; 4) a small posterior internal capsule infarction with transient unilateral hand numbness; 5 ) cerebrospinal otorrhea requiring a return to the operating room for repair; and 6) an intraventricular hemorrhage that resolved spontaneously. None of the 6 patients had long-term effects due to these complications. Only 2 $(1.3 \%)$ patients had a permanent neurological deficit that had not resolved at last follow-up. One patient had an immediate postoperative visual field deficit, and the other had a posterior choroidal artery branch infarction that resulted in unilateral hemiparesis.

\section{Discussion}

Various surgical approaches exist to treat MTLE. Despite established data on the ATL and transsylvian approaches, additional studies are needed to analyze seizure and neuropsychological outcomes after the subtemporal approach to MTLE. Multiple studies have compared outcomes between ATLs and selective amygdalohippocam-
TABLE 2. Engel classification outcome and antiepileptic drug use among patients who underwent sSAH with at least 1 year of follow-up

\begin{tabular}{cc}
\hline \multicolumn{1}{c}{ Variable } & No. of Patients $(\%)$ \\
\hline Engel class $(\mathrm{n}=152)$ & \\
\hline I & $88(57.9)$ \\
\hline II & $39(25.7)$ \\
\hline II & $15(9.9)$ \\
\hline IV & $10(6.6)$ \\
\hline Antiepileptic drug use & \\
\hline Among all patients $(n=152)$ & $23(15.1)$ \\
\hline No & $129(84.9)$ \\
\hline Ymong Engel class I patients $(n=88)$ & $23(26.1)$ \\
\hline No & $65(73.9)$ \\
\hline Yes
\end{tabular}

pectomies and their various approaches, but the number of selective amygdalohippocampectomies reported in such studies is low. ${ }^{2,9,14-16}$ This study evaluated seizure outcomes, neuropsychological outcomes, and complications in a large series of patients who underwent sSAH and represents the largest series of patients who have undergone this specific surgical approach published to date.

In this study, 152 patients had at least 1 year of clinical follow-up. Of these patients, 88 (57.9\%) demonstrated Engel class I seizure outcomes or freedom from disabling seizures, and $23(15.1 \%)$ patients were completely independent of antiepileptic drugs at the 1-year follow-up. These results are comparable to findings in the seminal trial by Wiebe et al. ${ }^{1}$ that compared ATL to medical management, in which $58 \%$ of patients in the surgical group demonstrated freedom from seizures impairing awareness at the 1-year follow-up. Our data must be interpreted cautiously because our study was a retrospective review of outcomes, whereas the study by Wiebe et al. was a randomized con-

TABLE 3. Engel classification outcome and antiepileptic drug use among patients who underwent SSAH with at least 2 years of follow-up

\begin{tabular}{cc}
\hline \multicolumn{1}{c}{ Variable } & No. of Patients $(\%)$ \\
\hline Engel class $(\mathrm{n}=124)$ & \\
\hline I & $70(56.5)$ \\
\hline II & $33(26.6)$ \\
\hline III & $13(10.5)$ \\
\hline IV & $8(6.5)$ \\
\hline Antiepileptic drug use \\
\hline Among all patients $(\mathrm{n}=124)$ & $22(17.7)$ \\
\hline No & $102(82.3)$ \\
\hline Yes & $22(31.4)$ \\
\hline Among Engel I patients $(\mathrm{n}=70)$ & $48(68.6)$ \\
\hline Yes
\end{tabular}


TABLE 4. Comparison of patient characteristics and Engel class I seizure outcomes among patients who underwent sSAH with at least 1 year of follow-up

\begin{tabular}{|c|c|c|c|c|}
\hline Variable & No. of Patients & No. of Patients (\%) in Engel Class I & OR $(95 \% \mathrm{Cl})$ & $\mathrm{p}$ Value \\
\hline \multicolumn{5}{|l|}{ Invasive monitoring } \\
\hline Yes & 63 & $28(44)$ & $0.387(0.199-0.753)$ & 0.005 \\
\hline No & 89 & $60(67)$ & & \\
\hline \multicolumn{5}{|l|}{ Sex } \\
\hline Male & 58 & $32(55)$ & $0.835(0.432-1.618)$ & 0.59 \\
\hline Female & 94 & $56(60)$ & & \\
\hline \multicolumn{5}{|l|}{ Side } \\
\hline Rt & 73 & $45(62)$ & $1.346(0.705-2.570)$ & 0.37 \\
\hline $\mathrm{Lt}$ & 79 & $43(54)$ & & \\
\hline \multicolumn{5}{|l|}{ MTS findings on MRI } \\
\hline Yes & 90 & $59(66)$ & $2.243(1.153-4.363)$ & 0.02 \\
\hline No & 61 & $28(46)$ & & \\
\hline \multicolumn{5}{|c|}{ Hippocampal sclerosis pathology findings } \\
\hline Yes & 141 & $80(57)$ & $0.437(0.0853-2.242)$ & 0.32 \\
\hline No & 8 & $6(75)$ & & \\
\hline
\end{tabular}

Boldface type indicates statistical significance.

trolled trial. Our results are also similar to those in another large single-center study that retrospectively analyzed patients who had undergone various surgical interventions for MTLE. Those authors concluded that the different surgical approaches analyzed resulted in similar seizure outcomes. ${ }^{2}$ Unfortunately, that study involved only 24 patients who had undergone sSAH.

The current study involved a large number of patients, and the findings indicate that sSAH compares favorably with traditional anterior lobectomy for the patients with Engel class I outcomes. Patients with Engel class I and II outcomes accounted for $83.6 \%$ of patients in this cohort, which indicates that, at 1 year of follow-up, the vast ma-

TABLE 5. Mean differences in preoperative and postoperative neuropsychological and neurocognitive measures among patients who underwent SSAH

\begin{tabular}{lcccccc}
\hline \multirow{2}{*}{\multicolumn{1}{c}{ Standardized Test }} & \multicolumn{2}{c}{$\begin{array}{c}\text { Dominant } \\
\text { Hemisphere }\end{array}$} & & \multicolumn{2}{c}{$\begin{array}{c}\text { Nondominant } \\
\text { Hemisphere }\end{array}$} \\
\cline { 2 - 3 } \cline { 6 - 7 } Verbal Comprehension Index & -1.27 & 0.48 & & 2 & 0.21 \\
\hline Perceptual Reasoning Index & 2.53 & 0.31 & & 0.06 & 0.97 \\
\hline Full-scale IQ & 1.21 & 0.13 & & 0.21 & 0.85 \\
\hline TMT part A & -0.33 & 0.85 & & -1.11 & 0.60 \\
\hline TMT part B & -4.29 & 0.05 & & -0.53 & 0.74 \\
\hline RAVLT-short delay & -7.21 & $\mathbf{0 . 0 2}$ & & 1.58 & 0.24 \\
\hline BVMT-R & -0.19 & 0.93 & & 1.39 & 0.46 \\
\hline BNT & -2.95 & $\mathbf{0 . 0 4}$ & & 2.22 & $\mathbf{0 . 0 4}$ \\
\hline BDI-II & -2.26 & 0.10 & & 0.33 & 0.79 \\
\hline
\end{tabular}

$\mathrm{MD}=$ mean difference.

Boldface type indicates statistical significance. jority of patients who underwent sSAH either were free of disabling seizures or had only rare disabling seizures. Moreover, the efficacy of sSAH seems to be maintained over time, as the percentage of patients who remained free of seizures was similar among patients with at least 1 year of follow-up and patients with at least 2 years of follow-up (Tables 2 and 3). Interestingly, skip candidates and patients with evidence of MTS were statistically more likely to achieve freedom from seizures than were nonskip patients or patients who did not demonstrate MTS on MRI. This phenomenon is likely since, intuitively, patients who are qualified to skip invasive intracranial monitoring should have a more definitive rationale for success after mesial temporal resection. Additionally, patients with MTS have radiographic evidence of the specific disease pathology treated with surgery, so the finding that such patients were more likely to achieve freedom from seizures also appears understandable. Patients who were deemed skip patients often demonstrated convincing MTS on MRI, with $85.4 \%$ (76/89) of the patients who avoided invasive monitoring demonstrating MTS on MRI. Because there are significant differences in seizure outcomes between patients with evidence of MTS on MRI versus those without, in the future, it may be necessary to consider nonsurgical options more readily for patients without MTS. Multiple nondestructive options, such as responsive neurostimulation or deep brain stimulation, are now approved and used throughout the country. It may be worthwhile to consider these options more keenly before permanently removing or ablating a normal-appearing hippocampus in patients without obvious MTS.

Moreover, there is growing evidence that stereotactic laser amygdalohippocampectomy (SLAH) results in similar seizure outcomes to those found in this study, with an even more minimally invasive approach. ${ }^{6,17}$ Similar to sSAH, SLAH leaves the temporal stem and anterotempo- 
ral lobe intact but requires a smaller operation. ${ }^{18}$ SLAH seems to impart some improved neuropsychological testing compared with traditional ATL. ${ }^{19}$ Analogous to the sSAH approach, SLAH does not preclude patients from further, more extensive resective surgery. ${ }^{17}$ Ultimately, long-term follow-up is needed to determine if seizure outcomes with ablative amygdalohippocampectomies are as durable as those with resective surgeries.

At our institution, patients with concordant data implicating the anterolateral temporal lobe as well as the mesial structures are recommended to undergo a standard ATL. Although our study did not directly analyze this patient population, invasive monitoring implicating an epileptogenic zone that also included the anterolateral temporal lobe was also an indication that they should undergo ATL. It is a limitation of our study that these patients cannot be directly compared with the sSAH patient population. It could be posited that some of the patients who were not Engel class I after sSAH had an epileptogenic zone that included the anterolateral temporal lobe spared by our technique. Additionally, of the 89 patients who went directly to surgery and skipped invasive monitoring, $89.9 \%$ (80/89) were Engel class I or II at 1-year follow-up. No skip patients with seizure recurrence went on to have invasive monitoring after sSAH. Two skip patients with seizure recurrence after sSAH went on to have a standard temporal lobectomy after further noninvasive evaluation was performed. It is possible that skip patients for whom sSAH failed had an epileptogenic zone that involved the anterolateral temporal lobe as well as the mesial structures.

Our study demonstrates that surgeons who have technical experience with the sSAH approach can achieve good seizure outcomes with a minimally invasive approach that leaves the temporal stem and anterolateral temporal lobe largely intact. There is a potential for a greater likelihood of seizure freedom in skip patients and patients with MTS on MRI. In addition, there was a 5.3\% (8/152) complication rate, $1.3 \%(2 / 152)$ permanent morbidity rate, and $0.0 \%$ mortality rate. These rates are low and compare favorably to those in several other large studies analyzing morbidity and mortality outcomes after surgery for MTLE.,

The anterolateral temporal lobe is not generally considered to be highly eloquent cortex, as resection does not result in overt and immediate postoperative deficits. Nevertheless, multiple studies have demonstrated the neurocognitive, language, and executive functions of the anterolateral temporal lobe, particularly on the dominant side..$^{20}$ When comparing preoperative and postoperative neuropsychological testing in this study, patients undergoing SSAH demonstrated no significant change in intelligence, verbal comprehension, perceptual reasoning, attention and processing, cognitive flexibility, visuospatial memory, and mood. Of note, a significant decline in word retrieval was found in patients regardless of the side of surgery, and a significant decline in verbal memory was found only in patients who underwent resection involving the dominant hemisphere. Declines in verbal memory have been demonstrated in multiple earlier studies examining patients who underwent selective amydalohippocampectomies, with worse declines affecting patients with dominant-hemisphere resections..$^{8,14,21-23}$ Our study suggests that there is still potential for a postoperative decline in language function with the sSAH approach despite the preservation of the anterolateral cortex and temporal stem.

Despite these findings, multiple other tests of neurocognition and mood did not detect changes among the patients in our study. It would seem intuitive that preserving as much functioning cortex as possible would result in improved cognitive outcomes; however, more robust direct comparison studies are needed that compare sSAH with complete ATLs to elucidate this relationship. It should be noted that many social and behavioral skills related to intact frontotemporal connections may not be fully evaluated by standard neuropsychiatric tests but are, nevertheless, worth preserving for optimal human interactions and employability. In addition, a study by Schmeiser et al. ${ }^{9}$ found significantly less frequent and less severe visual field deficits in patients who underwent sSAH compared with those who underwent ATL and transsylvian selective amygdalohippocampectomy. Although patients in this study did not undergo formal visual field testing, all patients underwent bedside visual field confrontation testing as part of their preoperative and postoperative neurological examination. In our entire cohort, only one patient demonstrated a postoperative visual field deficit. Although these results must be interpreted cautiously because bedside visual field testing is notoriously unreliable, the findings are compatible with one of the goals of the subtemporal approach, primarily avoiding the superoposterior temporal optic radiation tracts.

At our institution, with a single epilepsy surgeon performing all resective epilepsy procedures, sSAH is considered a first-line treatment option for MTLE. Additional temporal or cortical resection is performed only if significant preoperative workup or invasive testing demonstrates epileptogenic tissue in areas of the brain outside of the amygdala, hippocampus, and parahippocampal structures. The sSAH was originally proposed to preserve the anterolateral temporal lobe and the temporal stem ${ }^{24}$ while still achieving adequate deep epileptogenic tissue resection. Multiple additional techniques for performing a selective amygdalohippocampectomy have been described, including transsylvian and inferior temporal gyrus approaches. However, all of these procedures require additional cortical or white matter tract transection when compared with the subtemporal approach. ${ }^{25}$ The rationale for a subtemporal approach is that removing less cortical and white matter structure, as is the case with the sSAH, should be advantageous as long as seizure and neuropsychological outcomes remain equal or superior to other approaches. In addition, this sSAH approach is minimally invasive and requires only a 3 - to $4-\mathrm{cm}$ incision, minimal hair shaving, and an even-smaller 2 -cm craniotomy that rests well below the thick inferior temporalis muscle. All of the mesial temporal structures can be safely resected through this keyhole technique while still leaving a significant quantity of superficial cortex and the white matter tracts intact (Fig. 3).

This study has several limitations. The retrospective nature of the data can result in both observer and interpretation bias. In addition, because our study involves a single-surgeon, single-center series, our results may be difficult to generalize to other centers. Furthermore, there is no 

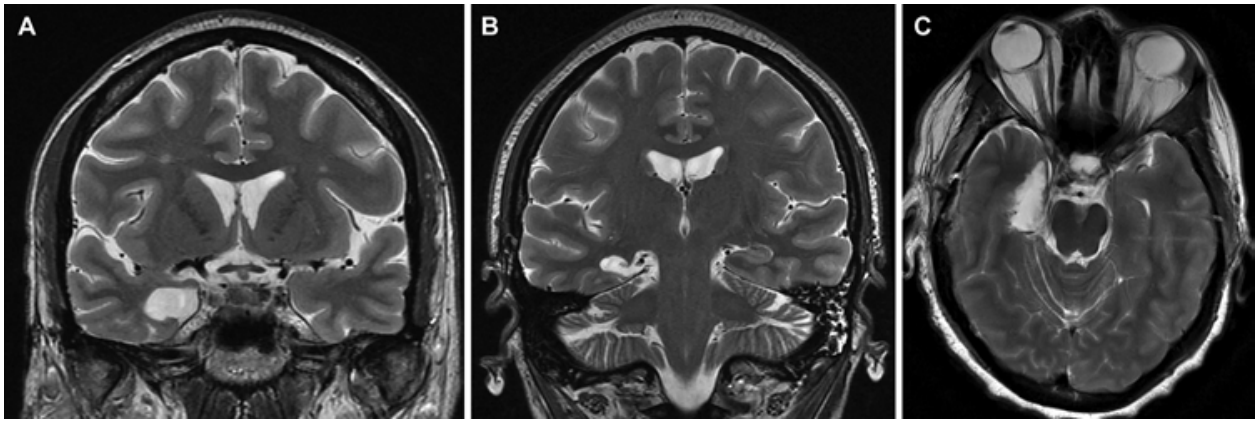

FIG. 3. Postoperative anterior coronal (A), posterior coronal (B), and axial (C) T2-weighted MR images demonstrating resection of the mesial temporal structures with preservation of the anterolateral temporal lobe and temporal stem.

comparison treatment group of patients with MTLE who did not undergo sSAH. Comparing outcomes between the sSAH approach and the ATL would have allowed more definitive conclusions to be made regarding the newer technique compared with the established approach with foundational evidence.

Although there were a significant number of patients with long-term clinical follow-up, a large percentage of these patients did not complete postoperative neuropsychological evaluations. There were several reasons for this, including challenges with insurance funding of postoperative neuropsychological testing, patient time constraints, travel for out-of-town patients, and lack of social support for others to make multiple medical follow-up visits. The small number of patients with neuropsychological follow-up reduces the power of the outcome results and raises questions as to whether definitive statements can be made about postoperative stability or changes in specific outcome measures. Even with little follow-up data, this study is still the largest series to date with neuropsychological outcomes after the sSAH approach and provides useful information regarding postoperative neurocognitive changes, although our findings must be interpreted with caution.

Finally, this study was limited by the follow-up duration. Although 1-year and 2-year seizure outcomes are frequently reported in the literature, they might not predict true long-term seizure outcomes and durability of the therapy. In the case of the sSAH approach, where more cortex and white matter tracts are spared, there is the potential for epileptogenic tissue to be left behind. This situation can result in declining rates of freedom from seizure over time compared with other approaches.

\section{Conclusions}

This study describes a minimally invasive, sSAH technique in a large series of patients with MTLE. Patients with at least 1 year of follow-up were found to have a $57.9 \%$ $(88 / 152)$ rate of Engel class I seizure outcome or freedom from disabling seizures. Preoperative and postoperative neuropsychological testing indicated no significant change in intelligence, verbal comprehension, perceptual reasoning, attention and processing, cognitive flexibility, visuospatial memory, and mood. Of note, patients did demonstrate a significant change in word retrieval regardless of the side of surgery, and a significant change in verbal memory was found among patients who underwent dominant-side resection. Complication rates were low, with a $1.3 \%(2 / 152)$ permanent morbidity rate and $0.0 \%$ mortality rate. Overall, the sSAH approach applied in this study indicates the safety of this minimally invasive technique for the surgical treatment of MTLE. The sSAH approach was associated with good seizure outcomes and a low complication rate. Neuropsychological outcomes appear to be comparable to those associated with other more invasive approaches.

\section{Acknowledgments}

We thank the staff of Neuroscience Publications at Barrow Neurological Institute for assistance with manuscript and video preparation.

\section{References}

1. Wiebe S, Blume WT, Girvin JP, Eliasziw M. A randomized, controlled trial of surgery for temporal-lobe epilepsy. $N$ Engl J Med. 2001;345(5):311-318.

2. Schmeiser B, Wagner K, Schulze-Bonhage A, et al. Surgical treatment of mesiotemporal lobe epilepsy: which approach is favorable? Neurosurgery. 2017;81(6):992-1004

3. King JT Jr, Sperling MR, Justice AC, O'Connor MJ. A cost-effectiveness analysis of anterior temporal lobectomy for intractable temporal lobe epilepsy. J Neurosurg. 1997;87(1):20-28.

4. Langfitt JT. Cost-effectiveness of anterotemporal lobectomy in medically intractable complex partial epilepsy. Epilepsia. 1997;38(2):154-163.

5. Picot MC, Jaussent A, Neveu D, et al. Cost-effectiveness analysis of epilepsy surgery in a controlled cohort of adult patients with intractable partial epilepsy: a 5-year follow-up study. Epilepsia. 2016;57(10):1669-1679.

6. Gross RE, Stern MA, Willie JT, et al. Stereotactic laser amygdalohippocampotomy for mesial temporal lobe epilepsy. Ann Neurol. 2018;83(3):575-587.

7. Le S, Ho AL, Fisher RS, et al. Laser interstitial thermal therapy (LITT): seizure outcomes for refractory mesial temporal lobe epilepsy. Epilepsy Behav. 2018;89:37-41.

8. Schoenberg MR, Clifton WE, Sever RW, Vale FL. Neuropsychology outcomes following trephine epilepsy surgery: the inferior temporal gyrus approach for amygdalohippocampectomy in medically refractory mesial temporal lobe epilepsy. Neurosurgery. 2018;82(6):833-841.

9. Schmeiser B, Daniel M, Kogias E, et al. Visual field defects following different resective procedures for mesiotemporal lobe epilepsy. Epilepsy Behav. 2017;76:39-45. 
10. Engel J. Surgical Treatment of Epilepsies. 2nd ed. Raven; 1993.

11. Altman DG. Practical Statistics for Medical Research. 1st ed. Chapman \& Hall; 1991.

12. Sheskin DJ. Handbook of Parametric and Nonparametric Statistical Procedures. 3rd ed. Chapman \& Hall; 2003.

13. Little AS, Smith KA, Kirlin K, et al. Modifications to the subtemporal selective amygdalohippocampectomy using a minimal-access technique: seizure and neuropsychological outcomes. J Neurosurg. 2009;111(6):1263-1274.

14. von Rhein B, Nelles M, Urbach H, et al. Neuropsychological outcome after selective amygdalohippocampectomy: subtemporal versus transsylvian approach. J Neurol Neurosurg Psychiatry. 2012;83(9):887-893.

15. Wang C, Liu D, Yang Z, Yang Z. Clinical outcomes after medial temporal lobe epilepsy surgery: anterior temporal lobectomy versus selective amygdalohippocampectomy. Zhong Nan Da Xие Xие Bao Yi Xue Ban. 2018;43(6):638-645.

16. Elliott CA, Broad A, Narvacan K, et al. Seizure outcome in pediatric medically refractory temporal lobe epilepsy surgery: selective amygdalohippocampectomy versus anterior temporal lobectomy. J Neurosurg Pediatr. 2018;22(3):276282.

17. Gross RE, Willie JT, Drane DL. The role of stereotactic laser amygdalohippocampotomy in mesial temporal lobe epilepsy. Neurosurg Clin N Am. 2016;27(1):37-50.

18. Jermakowicz WJ, Kanner AM, Sur S, et al. Laser thermal ablation for mesiotemporal epilepsy: Analysis of ablation volumes and trajectories. Epilepsia. 2017;58(5):801-810.

19. Drane DL, Loring DW, Voets NL, et al. Better object recognition and naming outcome with MRI-guided stereotactic laser amygdalohippocampotomy for temporal lobe epilepsy. Epilepsia. 2015;56(1):101-113.

20. Sherman EM, Wiebe S, Fay-McClymont TB, et al. Neuropsychological outcomes after epilepsy surgery: systematic review and pooled estimates. Epilepsia. 2011;52(5):857-869.

21. Yang PF, Zhang HJ, Pei JS, et al. Neuropsychological outcomes of subtemporal selective amygdalohippocampectomy via a small craniotomy. J Neurosurg. 2016;125(1):67-74.

22. Helmstaedter C, Richter S, Röske S, et al. Differential effects of temporal pole resection with amygdalohippocampectomy versus selective amygdalohippocampectomy on material-specific memory in patients with mesial temporal lobe epilepsy. Epilepsia. 2008;49(1):88-97.
23. Hill SW, Gale SD, Pearson C, Smith K. Neuropsychological outcome following minimal access subtemporal selective amygdalohippocampectomy. Seizure. 2012;21(5):353-360.

24. Hori T, Tabuchi S, Kurosaki M, et al. Subtemporal amygdalohippocampectomy for treating medically intractable temporal lobe epilepsy. Neurosurgery. 1993;33(1):50-57.

25. Alonso Vanegas MA, Lew SM, Morino M, Sarmento SA. Microsurgical techniques in temporal lobe epilepsy. Epilepsia. 2017;58(suppl 1):10-18.

\section{Disclosures}

The authors report no conflict of interest concerning the materials or methods used in this study or the findings specified in this paper.

\section{Author Contributions}

Conception and design: Smith, Whiting, Chen. Acquisition of data: all authors. Analysis and interpretation of data: all authors. Drafting the article: all authors. Critically revising the article: all authors. Reviewed submitted version of manuscript: all authors. Statistical analysis: all authors. Administrative/technical/material support: Smith, Whiting, Chen. Study supervision: Smith, Whiting, Chen.

\section{Supplemental Information \\ Videos}

Video 1. https://vimeo.com/402883530.

\section{Correspondence}

Kris A. Smith: c/o Neuroscience Publications, Barrow Neurological Institute, St. Joseph's Hospital and Medical Center, Phoenix, AZ. neuropub@barrowneuro.org. 